\title{
Une force, un objet, un champ : le buti des Kukuya au Congo.
}

(Magie d'agression et sorcellerie)

A Force, an Object, A Field: The Buti of the Kukuya (Congo): The Magic of Aggression and Sorcery

\section{Pierre Bonnafé}

\section{OpenEdition}

\section{Journals}

Édition électronique

URL : http://journals.openedition.org/span/1028

DOI : $10.4000 /$ span. 1028

ISSN : 2268-1558

\section{Éditeur}

École pratique des hautes études. Sciences humaines

\section{Édition imprimée}

Date de publication : 1 septembre 1987

Pagination : 25-67

ISSN : 0294-7080

\section{Référence électronique}

Pierre Bonnafé, "Une force, un objet, un champ : le buti des Kukuya au Congo. », Systèmes de pensée en Afrique noire [En ligne], 8| 1987, mis en ligne le 16 octobre 2013, consulté le 30 avril 2019. URL http://journals.openedition.org/span/1028 ; DOI : 10.4000/span.1028 


\section{ONE FORCE, ON OBJET, ON CHAMP : LE BUTI DES KUKUYA AU CONGO}

(Magie d'agression et sorcellerie)

par

\section{Pierre Bonnafé}

Les faits de magie d'agression et de sorcellerie, dont je désire parler, regardent la petite société des Kukuya : 12000 habitants aujourd'hui, compris dans l'aire culturelle du peuple teke. C'est une formation sociale lignagère, à droits partagés bilatéraux. Ses membres pratiquaient l'agriculture de savane, la chasse, la collecte, le tissage du raphia, la forge, sur les quelques arpents de leur plateau. La plus ancienne phase de leur histoire confrontait en une communauté hiérarchisée des "seigneurs de la terre" (mfumu a ntsie) à des lignages matrilinéaires (nzo) possédés par les premiers. A partir du milieu du $18 \mathrm{e}$ siècle environ, on assista à l'instauration d'une seconde couche dominante, "les seigneurs du ciel" (mfumu a yulu), qui mirent en place un système plus vaste et plus conflictuel: le mpu. L'ensemble constituait une aristocratie tributaire en voie d'étatisation, régnant sur des subordonnés (nkani) : femmes, clients, cadets, dépendants, captifs intégrés. Vers 1900, la région faisait transiter vers la mer lointaine ou dans d'autres directions du fer brut et des esclaves.

Malgré la rupture coloniale, aucun pan de cet édifice n'avait totalement disparu lors de l'Indépendance du Congo en 1960. Pourtant, les heurts de la conquête, puis de la pacification avaient disloqué le système politique, mis fin au commerce antérieur, avant d'imposer 
taxes, regroupements villageois et corvées. I'administration française ne réussit à établir son "ordre" qu'en passant des alliances avec des notables locaux à tous les échelons. Après l'édification de cette infrastructure, une seconde période, après 1930, put être corisacrée au développement des cultures marchandes et d'un microsalariat. De ce trop bref rappel, qu'est-ce qui touche le plus notre sujet ?

Les gens de la société colonisée réagirent par des tactiques variées : résistance passive au travail forcé, mouvement d'ouverture vers les villes, mais la région demeura quelque peu à l'écart, protégée tout un temps par ses mauvaises routes. Ce mélange de changements inoui's et de blocages politiques amena un lot de contradictions insolubles. Comme beaucoup d'ethnologues l'ont observé en Afrique noire après la seconde guerre mondiale, le culte des ancêtres périclita et la magie d'agression ainsi que la sorcellerie "envahirent le pays". Cette opinion était partagée sur place par bien des adultes des deux sexes. L'état de fait se prolongeait encore en 1965-67, date de ma première enquête. Toute la population distinguait aussi nettement qu'E. Evans-Pritchard, à la suite des Azandé du Soudan, entre une sorcellerie innée et organique ("witchcraft") et une magie d'agression périlleuse ("sorcery"), plus axée sur la manipulation d'objets (Evans-Pritchard, 1937: 9-11). Le premier pouvoir, ils le nommaient pfuna - l'autopsie ancienne "le" découvrait dans certains cadavres, - le second ils l'appelaient buti (au pluriel : mati). Les deux puissances étaient capables d'ensorceler (kiloko), c'est-à-dire de détruire la santé ou la vie d'autrui.

\section{A. L'objet-force buti}

Que aire au minimum d'un tel buti pour faire ressortir un ensemble de traits définissant sa cohérence? Les habitants au pays ne se soucieraient guère de lui, s'il n'était doté d'un pouvoir sur leur environnement ou sur eux-mêmes. Un tel objet est envié, redouté, acquis, transmis, renforcé, diminué ou détruit, parce qu'il agit. Pourtant, sa force n'est pas la force courante du corps: mpini, mais on peut lui conférer plusieurs noms : mpiini, la force 
transformée, mpani, la puissance invisible, kikini, le renfort. Toutes ces puissances ont en commun d'appartenir à un monde différent du monde "simple". Par conséquent, se défendre contre elles ou les acquérir pour les manier, suppose qu'on fasse appel à une relation cachée avec ce monde invisible. Les sens habituels ne suffisent pas à le percevoir : l'univers est inconnaissable par l'expérience ordinaire. Depuis sa création par Nzami, l'Etre suprême, ou autrefois Ngampu, on ne peut rien faire d'autre qu'éprouver le monde dans son flamboiement du soleil à midi ou dans son lever du jour. Le reste des enchainements demeure opaque.

Un homme nous racontait, il y a deux ans, son passage vers 1960 au catéchisme catholique : "Nous, enfants du pays, nous refusions l'idée de Création du monde à l'école des missionnaires. La Genèse? Comment inaginer une histoire d'avant les hommes ? On nous traitait d'athées! Nous, nous savions que Nzami existe par tout ce qu'on voit. En dehors de cela, nous ne cherchons pas à nous confronter à lui par d'autres moyens. Le reste est pure imagination. Nous, nous ne connaissions que les mati qu'on "excite" et "subit", parce qu'on en voit les effets." Il concluait avec I'humour macabre de son peuple : "quant aux saints, nous les tenions pour des criminels, tous comme tout le monde !" Cet appel à une expérience réaliste contre une métaphysique transcendante est très éclairant : la pureté des Saints n'y résiste pas plus que la virginité de la Vierge. Mais ces perceptions sont aussi conjoncturelles : à l'époque de leur éducation, bien des nouveaux fidèles se laissèrent persuader d'unifier en Diable leurs mauvais génies bapfu. A présent, une décennie après, le reflux oscillant des conversions ramène la dominance d'un champ magique si puissamment qu'on n'a jamais vu surgir ici le moindre prophétisme.

Tout ce qui précède pourrait se traduire presque mot-à-mot. Les deux grandes divisions de l'univers se recoupent avec celles du temps : mandaka ma wiri, "les affaires du jour" et mandaka ma mpiriba, "les affaires de la nuit", normal et déterminé contre anormal et indéterminé. Mais pour les habitants, visible et invisible s'entremêlent sans cesse quelque part un moment, sinon ils ne se soucieraient guère de leur relation à l'inconnu. Certains faits visibles sont 
perceptibles le jour soit directement par chacun, soit par l'expérience extérieure d'autrui ou de la nature - en cela, les gens ne diffèrent nullement de nous - et des individus les rapportent à des faits visibles, censés les expliquer ou en découler. A l'inverse, certains faits visibles sont à leur tour perceptibles par leur prolongement nocturne et ils sont référés à leurs connexions invisibles (ainsi d'une dispute ou d'une injure du jour arrivant jusque dans un rêve). Ces phrases ont l'air de s'appliquer parfaitement à d'innombrables cas de sorcellerie, que j'ai entendus avec leur déroulement... L'ennui, si l'on supprime la division jour/nuit, très spécifique par rapport à nos sociétés, est qu'elles sont universelles :

Quelle est donc l'originalité du lien caché entre une personne mburu et son objet buti ? Comment distinguer son activité de celle d'un savant moderne, d'un prêtre catholique, d'un prestigiditateur... ou même d'un homme kukuya sacrifiant le soir à ses ancêtres un coq avec des noix de kola ou du vin de palme?

\section{B. Présupposés et hypothèses}

1. La force d'une entité comme le buti, nous la considèrerons comme force sociale, par un raccourci: le mouvement de socialisation sera saisi à travers deux pôles opposés, qui sont l'individuel et le collectif. Le paradoxe est que cet abord direct, qui nous rapproche de celui des Kukuya, nous conduira immanquablement à des détours, vécus par les gens eux-mêmes, lorsqu'ils prétendent "détourner des vies" dans leurs objets magiques.

2. Cette force (ou rapport) sociale, nous la prendrons comme symbolisée sur deux plans distincts : celui de la logique des choses et celui de la logique des mots. Le premier relève de la matérialité objective, le second de la symbolisation subjective. Mais les envisager clairement comme distincts ne veut pas dire les séparer ! Tout notre effort va au contraire porter sur leur jeu d'interactions.

A la suite de F. de Saussure, on a pu donner une définition de Ia langue comme faculté de communication, émettant en droit 
tous les énoncés possibles ${ }^{1}$; mais on lui a opposé à juste titre le cas de l'idéologie comme l'unité de la pluralité contradictoire. Cet aspect unitaire non plus ne nous parait pas le plus important. E. Terray² a montré comment une formule comme celle de M. Augé : "toute organisation est en même temps représentation", jointe à l'abandon de la hiérarchie entre rapports sociaux, impliquait le choix d'un espace isomorphe, conception que nous récusons, nous aussi.

C'est pourquoi notre hypothèse sera inverse : la force sociale ne peut être que contradictoire, irréductibilité qui entraine un espace hétérogène et une hiérarchie du social. Mais s'il est vrai que "Ie langage reproduit toujours la réalité" (E. Benvéniste, 1966: 175), ne convient-il pas de pousser l'idée à son terme : le langage reproduit toujours les contradictions de la réalité sociale? Le concept de discours social nous servira de pont entre ces sphères, parce qu'il relève du langage et s'articule sur la société. Le lecteur sera d'avance convaincu que la réflexion sur ce thème ne peut avancer que par l'examen de faits singuliers !

\section{I - FORCE}

\section{A. Ia représentation de l'univers (mukiri)}

1. Grandes conceptions

Il faut bien poser au moins la dualité de l'univers pour concevoir comment des humains y agissent. Nzami a créé en même temps les hommes, les femmes, les animaux et les plantes ainsi que les éléments. C'est ainsi qu'il a pourvu les humains d'un principe vital mumpuki, lié à celui de leur lignage nzo, le mumpuki a nzo, et à celui de leur terre : le mumpuki a ntsie. Ces principes sont

\footnotetext{
1 Voir E. Terray, 1978: 128

20p.cit. 123-128. L'"idéo-logique" de M. Augé est "construite, voulue, consciente" (1975: 74). Comme E. Terray le note bien, l'unité dans le signe chez F. de Saussure est du côté du signifiant.
} 
des forces assurant le succès à tous : mpolo. Mais Nzami a créé simultanément des pfuna, qui sont les "choses" agissantes de la sorcellerie. Dès cette origine, la sorcellerie kiloko existait comme les sorciers humains.

L'important pour la santé, la prospérité mpolo, c'est d'avoir son mumpuki "en place" : mumpuki qa mbuka et tout réusit : enfants, semences... Une maladie déséquilibre le principe vital. On dit : mpolo ma fa mari mu nyuru, "la santé s'envole de son corps". Ce principe est lié à la source au lignage matrilinéaire.

De même qu'il y a diversité chez les humains, il y a diversité chez les animaux et les plantes, qui renferment des forces très différentes et inégales entre elles. La création a donné aussi aux hommes la faculté de "se composer" (kiwama) et d'acquérir les forces redoutables de la nature. I'homme s'est trouvé ainsi plongé dans un monde rempli de forces, dont certaines le traversaient, d'autres non. De toute manière, leur connaissance lui échappait. Par contre, ce monde était maniable, en ce sens qu'une partie des gens en savait long sur des fragments de ce savoir instrumental. Des privilégiés s'en emparèrent : les spécialistes ngàà, "ceux qui avaient le don", ainsi que des puissants (muwani). Les autres, pauvres hères, étaient peu concernés par leurs batailles, car, au milieu de ces rapaces, il valait mieux faire bonne figure en cherchant la protection de I'un d'eux.

\section{Savoirs}

Le savoir correspondant à de telles conceptions est cloisonné et partiel à la fois. Ses détenteurs ne mâtrisent que la puissance correspondante. Il est rare qu'ils en possèdent plus de deux ou trois principales. De plus, ce que dissimule souvent l'uniformité des transmissions (par exemple père-fils), elle reste secrète. Une puissance révélée à tous perdrait son efficacité. D'ailleurs, est-ce pensable? Ou on s'en sert ou on s'en débarrasse comme d'un déchet radio-actif. 
En résumé on rencontre trois couches de références lorsqu'on veut rendre compte de ce savoir :

- La création du monde relatée.

- La transmission de certains secrets "expérimentaux", formant autant d'objets partiels à tous les niveaux de la hiérarchie. En ce second cas, surtout si le statut politique est élevé, les détenteurs se réclament de leur lignée d'ancêtres pour les muer en "protections" religieuses. Les seigneurs du ciel sont enterrés dans des cimetières séparés de leurs subordonnés. Leur clan patrilinéaire a pour emblème la panthère. La conjoncture moderne a souvent mêlé tous ces types de pouvoirs par croisements divers.

- Infin, de nos jours, dans l'actualité d'un présent qui a toujours existé depuis des siècles, un personnage voulant "se renforcer", va être obligé d'acquérir une puissance et son savoir correspondant, bu-ngàà, chez un spécialiste. En ce dernier cas, toute la légitimité reste à conquérir ! Situation constante : le crédit de la corporation des ngàà fut reposé à chaque période sous un angle nouveau : autrefois, ils s'alliaient ou coexistaient avec les seigneurs ; sous la colonisation, ils dûrent se cacher ; à présent, ils tentent un compromis avec les autorités nationales.

\section{B. La classification des animaux, des végétaux et du reste de l'univers :}

On énumère toute une série d'animaux possédant une sorcellerie (kiloko) créée par Nzami. Caractéristique est leur prise de décision ferme et leur détermination à agir, si besoin est. Leur force (mpani) et leur cruauté (mputu) sont admirées par les sorciers qui s'en feront autant de défenses. Ainsi de l'aigle, de la panthère, du lion, du boa, de l'épervier, du caìman, du buffle, de l'éléphant, de l'hippopotame, du serpent mudzuma, de la biche nka, qui pille les cultures des femmes, des rongeurs mfie et mubaki, dévorant la volaille, au chat sauvage, comparé à la panthère. Tous ces animaux sont originairement des sorciers; une fois qu'ils tiennent une 
proie, jamais ils ne la lâchent ! Observant leur férocité, les sorciers humains ont supposé que silis réussissaient à les capter, alors ils pourraient vaincre même un homme invulnérable. Ils auront allié ainsi la compulsion à tuer des bêtes voraces à la leur 3 . Ces animaux sont la plupart du temps eux-mêmes des mauvais génies: bapfu (sing. mupfu). Le désir effréné de les utiliser sans limite est reporté sur le sorcier, mais leur prodigieuse force est tout de même admirée de presque tous.

D'autres sont puissants d'une autre manière comme mbumburu, la mère des termites, apte à se reproduire et à durer ; comme le petit rat ntsika, habitant souterrain d'une demeure pourvue de fenêtres, ne sortant jamais par la porte, fuyant par les ravins.; comme le lézard nganguru, qui change de couleur à l'occasion, et de nombreux animaux du même genre. Ainsi le serpent muyini, qui demeure dans les nuages, indénichable, sauf s'il pleut, semble-t-il. Le crapaud kiwuku est un mupfu, jamais consommé par les gens, qui peut s'introduire dans les maisons sans qu'on y prenne garde.

On complètera la liste des forces naturelles par ompfu nzwunu, le génie albinos, devenant la nuit un vrai européen : on ne peut lui rendre ses coups... et encore dzandale (= phonétiquement "gendarme") : "clest la même chose que l'européen".

Il n'y a pas que des animaux, des végétaux ou de nouvelles forces historiques. D'autres formes recouvrent des entités voisines, mais différentes : la force du ravin permettant de se cacher, celle du marché de se perdre dans la foule. "Autrefois, les gens se métamorphosaient en buisson, sillon, arbre. On peut abattre l'arbre et l'homme est parti depuis longtemps !" Ce sont plutôt des utilisations vécues de l'espace de la savane ou des lieux fréquentés selon un mode propre de projection-identification. Tantôt on "se change en", tantôt on "s'introduit dans".

3"La rigidité d'un mur, la détente d'un jaguar, la puissance d'un taureau, la force d'un éléphant, l'agilité d'un singe, la férocité d'un tigre, la patience d'un chat...", voilà les qualités que le Journal du Dimanche (26 septembre 86 ) souhaitait à l'équipe de France de volley-ball. 
Pareille classification repose sur la croyance préalable en un monde interactif entre humains et univers d'une certaine consistance. On n'en attend ni révélations multiples, ni fondations sacralisantes. On sait seulement qu'il agit. Il n'est pas d'une étoffe foncièrement différente de celle des humains, qui ont eux-aussi sorcellerie innée et capacité d'acquérir des mauvais génies. La voracité règne d'un bout à l'autre du monde, de même que la capacité de se défendre, la mort, la vie, la maladie. Mais au départ, de plusieurs manières (à l'origine, dans une période historique, dans une génération ou dans une vie humaine), ce lien virtuel reste invisible. Un homme ordinaire, une femme simple, un enfant non jumeau ne voient rien. C'est pourquoi cette similitude et cette latence renvoient à un lien caché avec la nature. Le mettre à jour nous éclairera sur le type de force utilisée.

\section{L'image du buti et celle des autres forces}

1. Caractéristiques de l'ensemble des forces

Le jeu des forces est dialectique et discontinu. Les éléments de l'univers se décomposent en puissances bonnes ou mauvaises qui s'opposent : la cruauté, le désordre et le succès, la prospérité; le principe vital humain et la sorcellerie; les gens ordinaires et les sorciers ou les grands contre-sorciers, les fauves et les animaux paisibles ; les mauvais génies et la santé. On nous a donné I'équivalent d'un ensemble de particules fastes ou néfastes (peut-être sont-elles le positif et le négatif d'un monde sans zéro!) : la mort est d'un côté, la vie de l'autre. Mais cette position de l'univers est globale : elle indique les grandes tendances possibles, révélées par la constitution du monde, sa formation avec tout ce qu'il faut virtuellement pour faire des équilibres ou créer des catastrophes. Jamais nous ne sommes descendus au quotidien des choses, bien qu'elles puissent s'insérer dans ces catégories.

On aurait tort de mésestimer leur profondeur. Je n'en offrirai qu'une illustration, non des moindres. L'Etre suprême, Nzami, malgré 
le peu d'explicitation qui nous en est fourni, est bien plus qu'un simple repère insignifiant. Il joue un rôle fondamental dans l'engendrement de l'être. Très comparable au mouvement uniforme découvert par Galilée, puis repris par Newton4, qui permet de penser le repos d'une pierre autrement que comme immobilité, il inscrit le cours régulier des jours, des nuits, des vies et des morts naturelles (de vieillesse en pleine santé), la poussée non contrariée des végétaux. Et n'a-t-il pas fallu l'indication de ce mouvement pour que soit concevable une poussée dans un sens ou dans un autre afin de maintenir une existence ou de la briser ? Mais le jeu politique est impensable dans ce détour premier. Aussi ne faut-il pas s'étonner de rencontrer des allusions à ce domaine dans tous les moments forts d'un rituel, le moindre proverbe sur les jumeaux ou la chasse.

Quels sont les éléments de cet univers ? Toute une série d'âmes ou de principes vitaux, dont l'essence est lignagère et le siège corporel sont confiés aux chefs lignagers, aux seigneurs de la terre, puis assujettis aux seigneurs du ciel. C'est bien l'image de la force sociale, construite à travers les siècles, qui nous est parvenue, avec sa double ligne de "possession" et de "direction". L'idée d'un monopole de la captation est au coeur d'un système tributaire, quoi qu'il arrive, surtout sill comporte un versant esclavagiste. Seigneurs et ainés n'ont pu que conquérir les forces de leur époque, investies dans la chasse, la cueillette, l'agriculture, le tissage et la forge. La conception de la force fut inégalitaire et anti-communautaire : les inégalités naturelles expliquaient et justifiaient indistinctement celles de la société. Telle en fut $\mathrm{du}$ moins la dominante, assez influente pour imposer à presque tous comme idée hiérarchique l'inégalité5.

4"La conclusion de Calilée, qui est correcte, a été formulée une génération plus tard par Newton comme la loi de l'inertie... Tout corps persévère dans son état de repos ou de mouvement uniforme en ligne droite, à moins qu'il ne soit déterminé à changer cet état par des forces agissant sur lui." Einstein (A.) et Infeld (L.), 1983: 12 .

5iautres peuples, comme les Lobi du Burkina Faso, associent la hiérarchie à l'égalité dans une société acéphale. 
2. L'image de force buti résulte des représentations sociales de la force.

Une semblable idée de la force sociale n'imprègne-t-elle pas celle des mati ? L'idée de "composition" (kiwama), centrale, en découle. En effet, elle renferme la notion d'une force ordinaire transformée (mpiini), avec pour fin principale de se distinguer de l'humanité courante. Elle est presque le corollaire de l'inégalité relevée.

La hiérarchie accomplit pleinement l'univers : beauté, succès, santé, fertilité... enfermées dans des corps. La sacralisation est à son apogée pour un seigneur du ciel. Les principes cités relient ces corps à la nature.

Mais en même temps, aspect beaucoup moins mis en relief (car on se contente souvent de montrer l'inversion), la hiérarchie tend $\grave{a}$ entraver cet accomplissement. C'est par ce moyen que s'instaure le contrôle ou le monopole des forts sur les identifications courantes d'un tisserand, d'une agricultrice, d'un chasseur ou d'une collectrice de sauterelles ou de chenilles. L'idée d'un hibou, attendant sa proie, chutant silencieusement sur elle et la dévorant traduit bien la situation induite sur un sujet.

Pourquoi avoir tenté de restituer l'atmosphère plus vaste des idées et sensations (mais les sensations ne sont-elles pas aussi des actions ?) entourant celles des mati ? Parce que cet imaginaire social est l'indispensable source où les groupes - lignages, hameaux, Terres - se socialisent entre les deux pôles de l'individuel et du collectif.

Ne se dégage-t-il pas de cet ensemble une idée propre de la force sociale? En effet la création de l'univers évoque un fait accompli, qui n'atteste pas seulement la reconnaissance lucide de la naissance simultanée de la bienfaisance et de la nocivité. D'abord, la puissance habite le corps de certains êtres, dont les humains. Mais surtout, d'emblée, l'écart est creusé : rapt, violence, on pourrait presque dire : "coup de force" ! Ici, comme dans toute 
l'aire teke, le "clinamen"6 est politique7, s'il faut entendre par là avec Lucrèce cette infime déviation, qui permet de mouvoir le monde.

A mieux préciser, on formulera ce saut initial comme politicomagique ou politico-religieux, sachant bien que ces domaines communiquent. Par voie de conséquence, les puissants s'étant révélés capables de faire main basse sur certains pouvoirs, les faibles se trouvent rejetés dans les ténèbres extérieures ou, si l'on préfère, la pénombre de l'humain ordinaire. Toute l'admiration va aux autres, sentiment qui est loin d'avoir disparu aujourd'hui, même s'il se transpose. Le résultat est ce que j'ai appelé naguère "la violence naturelle"18 (1970: 97-166).

Les résistances au pouvoir suscitent des idées opposées. Leur présence explique pourquoi dans le domaine des mati, les femmes sont vues comme mauvais génies femelles 9 , les cadets comme rebelles er puissance. En outre, les lignages ordinaires ont su endiguer les abus seigneuriaux, si bien qu'un courant communautaire est perceptible dans la pratique.

Ia représentation de la force sociale (réelle et imaginaire) ${ }^{10}$ paraît bien avoir entraîné celle des mati, comme un de ses cas particuliers.

6De nature rerun.

7 Le très riche livre de Jan Vansina (Tio Kingom of the great Makoko) retrace un tableau d'ensemble des Teke.

8 Cette notion m'a paru valoir pour analyser un grand rituel funéraire de seigneur du ciel : le miyali (1970: 97-166).

9Il y a un renversement : les femmes, plus faibles dans la vie courante et la puissance diurne, deviennent les plus redoutables des génies cachés.

10I'étude la plus fouillée sur cette question chez un peuple teke est celle de M.C. Dupré sur les Tsaayi, 1984, vol.I à propos du masque kidumu. Elle offre une excellente base comparative et tire des conclusions très proches des miennes. 


\section{OBJET}

\section{A. Un enjeu dans les rapports sociaux}

\section{Le cadre}

Un gros plan à présent, après ce long trajet. La vue se resserre: quelques acteurs, une assistance réduite à des gens qu'on peut toujours compter, si l'affaire est banale. Elle crôttra au contraire, quand l'incident sera grave. L'atelier en sera une ou plusieurs maisons, sa surface quelques hameaux. Les rebondissements pourront gagner une partie de Terre de quelques kilomètres, la rumeur s.'emparer d'une Terre quatre à cinq fois plus grande. Il faudrait un cataclysme pour rassembler plus de mille personnes. Bien que de multiples tentacules, encore aisément dénombrables, puissent partir vers les dix Terres de la région, et même en ville, le périmètre demeurera modeste.

Tout dans les pratiques y sera incroyablement concret. On ne parle pas de l'eau en général pour faire un rituel, soigner un malade, mais de telle eau, de telle mare avec un nom : celle-là et pas une autre. Je ne saurais mieux introduire à cet objet, apparemment fait de recettes passe-partout : la fameuse "cuisine magique", dont parlait M. Mauss. Jamais, je n'ai assisté à une action notable, qui n'implique des gens définis, des individualités et des groupes situables et une circonstance à sa manière unique. Même la routine y prend parfois ce visage.

L'expérience m'a conduit à penser cet objet buti en termes de force sociale. Plus les habitants me sont devenus proches, plus j'ai senti que l'essentiel se jouait dans cette dimension. Tel jeune homme allait consulter un devin en compagnie de son père, son grand-père, son oncle utérin et quelques parents des deux sexes, le soir, la nuit tombée. Telle femme, convoquée parce qu'elle était stérile, allait de guérisseur en guérisseur pour en trouver la cause, suivie par son époux, ses parents, parfois son seigneur de la terre. Tel décès brusque d'un homme affolait le voisinage, provoquant l'appel aux autorités supérieures : chefs, juges, contre- 
sorciers. Partout, une oscillation entre l'apaisement des conflits et leur mise en exergue, du quotidien usé à l'insupportable rapport de force avec un ou plusieurs perdants (1978: 8).

\section{Les trois niveaux}

Les rapports sociaux des années 1965 perpétuaient formellement une situation bien plus vieille. En bas, un système domestique avec ses petites maisonnées assurait la survie journalière. Des chefs de hameau et de foyer le dominaient en exploitant des femmes (35 heures de travail par semaine aux hommes actifs contre plus de 60 aux femmes). Des cadets souvent oisifs contribuaient aux revenus marchands.

Au milieu, une organisation lignagère permettait à une couche d'aînés et de notables une surextorsion importante sur le système domestique. Forte polygamie et forte dot combinaient leur poids pour opprimer femmes, cadets, paysans pauvres, et pousser ces dépendants à l'émigration.

Au sommet, un système extérieur marchand et souvent capitaliste, avec des classes nationales et étrangères, dominait et exploitait les précédents, en bénéficiant de leurs denrées commercialisables (tabac, pommes de terre, haricots). A cette date, seuls les hommes pratiquaient ces cultures, les paysannes servant de rente vivrière et domestique à tout le monde.

Les deux premiers niveaux ressemblaient à ceux de l'époque précoloniale, à la f'in, malgré toutes les vicissitudes de notre siècle. Le troisième, bien que comprenant l'Etat moderne, pouvait être comparé au système tributaire ancien. Du point de vue local, si capital politiquement, la petite société rurale était encore dominée par le système ligrager, nonobstant l'avancée de l'économie externe.

Dans cette configuration, à minuscule échelle, un objet magique buti va représenter un enjeu entre ces ainés, ces paysans pauvres, ces paysannes assez égales dans le dénuement, leurs enfants. Beaucoup d'affaires les concernant se règlent encore sur ce mode. Univers de séparation, ò̀ chacun agence son destin en faisant appel à un 
certain nombre de personnages : chef, supérieur de lignage, père et grands-parents, parents des deux sexes, alliés matrimoniaux, juges-arbitres et spécialistes (contre-sorciers, guérisseurs, devins, visionnaires...). Mort, maladie, santé, richesse, misère, fécondité f'éminine, espoir de bien se marier, chance de travailler et succès tout courts sont encore tramés à travers une culture - et son cadre politique -, où les entraves à la vie naturelle sont causées par des sorciers. Pas d'autre solution que de s'en défendre, même si on use des moyens si rares: école et dispensaire. Le reste est lointain comme le Gouvernement.

\section{Les consultations de magie}

A l'époque, comme par le passé, "les femmes n'ont pas plus de mati", qu'"elles n'ont de hameaux !" Qu'elles aient recours à ceux de leurs frères, époux, parents, ainés, semble normal. Plus tard, entre 1970 et 1985, leur mince accès à I'argent leur permettra d'en acquérir quelques-uns. Mais la supériorité des hommes se maintiendra avec une frontière floue.

Au surplus, la corporation des ngàà ne fut jamais exclusivement masculine. Quelques brillantes exceptions ont toujours confirmé la règle, soit par l'héritage d'un buti après décès, soit par son acquisition après une maladie. Mais des hommes disent : "une femme peut bien avoir de la science, plus qu'un homme même! Il lui manquera toujours lisi, la 'prestance', l'autorité visible du jour. Par contre, n'importe quel homme va tracer un simple trait mpiri (au kaolin) sur son front pour défendre un parent malade contre les ennemis". C'est une véritable objectivité imaginaire du "phallus" social, se déplaçant à travers les âges et changeant pour assurer sa durée.

Une spécialisation masculine a coïncidé, deux siècles durant, avec les activités créatrices de biens de prestige ou de valeurs marchandes. Ce quasi-monopole de la gestion sociale chez les aînés, les seigneurs, les notables, se révéla aussi complet que celui de la guerre autrefois, de la vengeance et de la chasse. Il entraîna la domination de tout un sexe sur l'autre. 
Ainsi, un front commun d'alnés, de riches, de chefs, de seigneurs se déploie face aux femmes, aux cadets et aux dépendants pauvres. Cette couche s'est appuyée sans cesse sur des juges-arbitres (ba wuobi) et sur des spécialistes de magie (ngàà). Le flux de l'argent est venu envahir le champ, orientant leur pouvoir vers les gains des paysans. Ces tendances vont se retrouver dans la galerie des objets puissants.

\section{B. Rites et croyances magiques}

1. Ia "composition" d'un buti (kiwama) ou sa "prise" (kitoko)

a) Description

Au sommet de la hiérarchie des forces naturelles, on rencontre le caiman, le buffle, la foudre (mvula ku yulu), l'hippopotame... On dira du premier comme de tous : ngandu ya ompfu : "Le caïman a un mauvais génie mupfu". Vouloir les acquérir auprès d'un ngàà réclame donc un véritable pacte : "pour les avoir, il faut faire mourir les personnes qui deviendront des bapfu elles-mêmes et vivront dans ce caïman ou dans cette panthère".

Certaines "compositions" (mpiini) exigent 3 ou 4 personnes, d'autres 27 comme le "boa de compétition", mbomo wu mikeme. Le plus souvent, le futur puissant doit partager avec le ngàà les personnes qui sont tuées et "détournées" dans leur arsenal invisible. Ce sont des parents très aimés qui seraient requis de préférence. La composition d'une telle puissance représentera le passage d'une force naturelle (mpini) à un produit transformé.

Un homme désire-t-il obtenir une puissance de vision (limuni)? Il devra réunir les signes de l'étoile (?), des plumes du petit oiseau lintoli, la peau de ntsi, un petit animal proche de la fouine avec des taches, ressemblant un peu à la panthère (I'animal repère les ennemis avant qu'ils n'attaquent par sa faculté de changer de taches: kikalika kele), un petit rat limpfina, le boa. Des fragments de cette force seront rassemblés, broyés. Le ngàà y mêlera des bouts d'ongles ou de cheveux du postulant. Il lui aura indiqué les interdits (ngili) 
propres à la puissance. Il crachera (kiseme) des ingrédients biseme, qui sont presque toujours identiques, très répandus au Congo ${ }^{11}$. Puis, il fera avaler cette préparation au candidat. Enfin, il lui ordonnera de ramasser les feuilles et tous les débris qui ont servi durant la cérémonie. Sur les feuilles étaient posés les morceaux du buti. Selon des modalités variables, le ngàà lui dira d'aller les jeter dans la rivière Mpama, voisine du plateau kukuya, ou dans la terre ou dans une termitière. Ces feuilles vont devenir mpiini en "se déformant" (kibalika = "changer de route, dévier"). Ce peut être encore à Brazzaville ou à Pointenoire ou la mer ou dans le fleuve Congo. Ce mpiini se métamorphose en Iion ou en mauvais génie selon une oscillation très remarquable. On voit que le résidu revient toujours à la "brousse" (onlie), lieu de plein exercice de sa force.

Voici réalisé à présent cet ensemble personne(mburu) - buti reste qu'on peut appeler mpiini ou encore la "chose" d'ur puissant muwani. Il est évident qu'il ne convient pas seulement de le considérer de son point de vue, mais d'imaginer l'effet qu'il déclenche sur des égaux ou sur des inférieurs. Nous aurons alors la "composition" en action. Prenons un spectaculaire exemple avec lifuru li tama : "la cenỏre de la minuscule grenouille tama", dont un possesseur nous a indiqué pourquoi il l'avait prise. "Voyant qu'il était haï par les membres de son lignage utérin (nzo) et de son lignage paternel (nzo tara) ainsi que par ceux du dehors, il partit voir un spécialiste pour l'acquérir. Le ngàà la lui fit rapporter d'une mare et lui composa sa puissance avec ses interdits propres. En mourant, il voulait dire à son fils: 'ne me pleurez pas sur le champ. Exécutez d'abord mes prescriptions ! Et le soir, vous pourrez pleurer'. Le tama, après sa mort, devait pénétrer dans les ventres de tous ceux qui avaient mangé leur père et les faire enfler. Les figures, les pieds, les bras, le dos. On devient informe, comme un rond. Quand le fils

${ }^{11}$ Les plus utilisés sont : nyimi, liyali (Guttiferes), nkere (Aracées), nduni (Moracées) et nzwu a nkwuna (Zingibéracées), tous végétaux. Le dernier est un piment sauvage : "si tu en prends, le buti de ton ventre se réveille et il devient comme une personne qui a perdu conscience" (Buti bu mu muin bu siliki, nde yiki wuri mburu wu bvi kitswe). On use aussi du poisson ngoli. 
verra les gens atteints de ce mal, il connaitra les coupables de la mort de son père. Il dira : 'on verra ''. Ceux qui ont gardé la chair quelques jours endureront la maladie un certain temps. Parfois, on peut même les opérer à l'hôpital. Ils guérissent. Demain, le tama les grossira encore. Il leur fournira une fois de plus son eau. Impossible de se rétablir. Ils mourront tous. Le possesseur de tama est très dangereux. On redoute d'avaler une telle personne. On ne le ferait jamais, si on le savait d'avance !".

b) Contenu des puissances

Des révélations de devins nous ont souvent appris le contenu des puissances "composées". Ainsi en 1967, I'un d'eux annonça à un habitant d'une Terre, venant de perdre un enfant, que son sorcier disposait de plusieurs mpiini : un éléphant, un caìman, un rat nkfumi, un serpent mubika également souterrains, un avion, une micheline (mikado), plus un autre dans la mer. Il faut réunir un morceau de toutes ces choses 12 , les donner au ngàà, qui les placera dans son fusil pour tirer. On nomme encore biseme (comme les ingrédients à cracher sur l'objet) tous ces fragments de vie. On doit les anéantir tous pour tuer le sorcier. Après blessure ou meurtre, tous ces mpiini deviennent visibles, car le principe vital du sorcier est faible. Un chasseur peut passer alors, par exemple, et lui tuer ses animaux.

Un maxinum de terreur est concentré dans certaines forces comme l'ompfu wu mbiele : le génie du couteau. Il faut pour l'acquérir des cheveux du fantôme kidzulu, pris au piège par des ngàà spéciaux, des cheveux de chimpanzé, complétés par d'autres biseme. Le détenteur, quand il l'a eu, met à mort une personne. Celle-ci devient son mupfu d'une extrême cruauté, parce qu'il n'entraîne pas une déperdition de force latente, mais un meurtre soudain et délibéré, par ailleurs aveugle. Un peu moins redoutable est le chef d'une bande wabi de génies faits pour amasser des richesses. Lui seul est chargé d'attrapper les gens pour les mettre à mal, les autres, ses compa-

120 bien de ce qui a été en contact avec elles : terre de l'aérodrome, bois d'une traverse de rail. 
gnons, ne font que les paisibles tâches de transport. Parmi cette équipe (qui ressemble aux anciennes caravanes de commerce!), un paysan, une paysanne peuvent avoir la malchance de rencontrer un parent "détourné" et de se faire voir de lui (un frisson l'indiquera...) ; ils risquent de périr si le génie les "effleure".

L'objet magique peut se résumer en un ensemble de normes ou d'interảts, s'appliquant à des occurrences. Le thème serait inépuisable ! Contentons-nous de dire qu'il convient de réactiver en permanence la vitalité de son buti. Il faut sans arrêt l' "exciter" par des offrandes, des incantations, des prescriptions particulières. "On ne nourrit pas ces puissances, mais on les excite", on leur "donne de la force" (kiwa mpini, avec un "i" bref, donc avec le sens de force physique) au moyen de noix de kola crachées et de vin, ou encore de sang, comme on en offre aux ancêtres. Le buti se manifestera parfois en noircissant pour prédire un malheur à son mâtre.

2. "L'excitation de la personne" et la "transformation de son corps".

Face à ces traductions littérales d'expressions kukuya, je préfère parler de "mise en état du sujet" et de "recoupement parallèle de son corps"13.

\section{a) Les expériences corporelles}

Il parât logique de partir de toutes les expériences concrètes qui font appel au registre corporel, parce que ce sont elles qui témoignent le mieux de cette "excitation de la personne", simultanée à celle de son buti, tant chez le puissant que chez le spécialiste ngàà. Il faut se donner beaucoup de mal pour devenir "l'animal ou la chose, dont on a pris la partie !". Attacher ses ongles, ses cheveux, qui une fois enterrés deviennent mpiini (force transformée), se dit kikura nyuru : "attacher son corps". Comme une chose ne

13Chez J. Lacan (1966: 871), on trouve une extrême attention au langage du sujet et du corps, dont j'essaie de tirer parti pour le sujet social, entre ses deux pôles individuel et collectif de socialisation. 
peut valoir qu'en entrant dans son ventre (mu muin), s'il veut l'utiliser, il doit manger les biseme prescrits par le spécialiste et les cracher sur le buti extérieur qui le représente. Ce dernier objet n'est qu'une "cage", permettant d'établir le circuit actif allant de la nature au corps. A chaque fois, donc, il conviendra de manger des biseme afin que le mpiini entende ses paroles. In magicien m'a même dit en parlant d'un faiseur de pluie : pendant qu'il fait tous ses gestes habituels, "sa sorcellerie (kiloko) entend". Un agresseur, fondant sur un puissant, n'a soudain plus affaire à la personne (mburu), mais à "sa sorcellerie". En effet, sa personne s'est "fortifiée ou renforcée".

Un ngáá-mbomo, "possesseur de boa", même s'il a eu la tête coupée, ne se putréfie qu'en partie. Son boa a déjà perdu sa queue ou sa tête. Pour périr complètement, sa respiration doit s'arrêter et on dit qu'il a 9 coeurs. Tous ces coeurs doivent "s'étouffer". Il faut savoir que pour un Kukuya le "coeur respire": ontima kafiere. De l'homme à l'animal se révèle une identité d'organes et de fonctions.

Les images employées vont des "racines" (midza yi mburu) de la personne à la maison invisible, gardée par une escorte de génies ou d'animaux cachés. Si l'on y réfléchit, c'est bien à partir d'une personne imaginée de la sorte que le domaine d'un seigneur de la terre ou surtout du ciel se retrouvait "barré", "enchevêtré" d'interdits (mikele mi ntsie) à lever en allant s'adresser spécialement à lui pour le supplier. Il fallait reprendre chez lui les semences et les outils qu'il avait dérobés mystérieusement. On dit aussi que le puissant tout entier est un arbre gigantesque.

Néanmoins, on doit prendre garde à la complexité des attitudes. On affirme souvent que le sorcier laisse son corps dormir à la maison, pendant qu'"il va nuire". Mais les termes dédoublés ne sont pas conçus comme étanches, comme le prouvent des phrases comme: ma ndaka ma kidzura muloki nyuru : "je vais chercher des moyens pour que le sorcier retire son corps!". Les gens parlent même parfois de forces innées pfuna (witchraft) supplémentaires. Ce sont elles que le détenteur ou le spécialiste ont l'air de recracher sous forme de biseme sur l'objet. 
b) Cycles humains et naturels

D'autre part, un énigmatique proverbe affirme : kibaka wa yala ("tout ce que tu gagnes, donne-le au tas d'ordures"). Yala, ce sont les ordures, y compris les excréments. Un peu semblable au fumier de Job dans la Bible, le yala proche du hameau finira par tout recueillir, y compris les puissances. On dit d'ailleurs que "yala est la mère du buti". Il faut comprendre : "à manger seul, on ne parvient qu'à ce tas a'ordures qui ne vous rapporte rien". c'est pourquoi il faut partager tout. On a là une très forte critique de la rétention, de l'avarice face à la mort.

Mentionnons pour clôre ce chapitre l'extraordinaire puissance de l'éléphant de semences nzoko a sala, qui avale les semences d'une autre Terre pour venir les déféquer dans la sienne. Ces cycles fondamentaux donnent une idée des affects en jeu, quand un chef de lignage, un seigneur de la terre ou du ciel disent encore : "j'ai mangé cette personne: elle m'appartient !", définissant ainsi la possession rituelle reliée aux corps et à la nature.

c) Le recoupement du "corps" parallèle à "l'excitation" déclenchée.

Toute une gamme de signes corporels révèle les émotions marquant le contact avec l'invisible. Un frisson (linzwumi) signale à quelqu' un I'approche d'un mauvais génie, un évanouissement 14 (kidzua ou kibva kitswe) indique plusieurs faits qu'il convient de démêler. Le même mot s'applique à la "perte de connaissance" (kibva kitswe) du ngàà, qui entre en transe. Dans les grandes occasions, les activités des contre-sorciers et des devins sont accompagnées par toute une mise en scène collective, à public restreint la plupart du temps. C'est ainsi que la nuit, on "excitera" un ngàà venu bravement délivrer son malade à l'agonie, quand tous les autres fuyaient. Lui, au contraire, est venu administrer sa "preuve" (mbiki). L'assistance l'aura encouragé à sortir de lui-même pour faire face au combat, elle lui aura offert ce qu'il voulait : un cabri, un coq, un pagne de danse (kiko ki ngàà). II s'en sera vêtu pour se surpasser. Souvent,

14 kidzwuà a vec un ton bas, signifie : tuer, avec un ton haut kidzwuá: s'évanouir. On doit distinguer aussi ngáá : "possesseur de" et ngàà : "spécialiste". 
les amis du malade le poussent à danser en l'insultant : "es-tu un ngàà véritable? La chose de ta mère?" (on ne dit rien sur le père, qui est pourtant le plus grand transmetteur de puissance). Furieux, il oubliera le danger. Notons qu'on peut injurier aussi son buti pour les mêmes raisons. La liste des injures graves comprend entre autres : sorcier, mupfu... montrant bien l'ambivalence des notions. Les ancêtres d'autrefois, quand on les injuriait jadis, rentraient chez eux et faisaient un serment appuyant leur vengeance.

Toutes les actions d'un puissant ou d'un ngàà dans les maisons ou les villages (écraser des pâtes mieme, préparer les macérations buobi ou des infusions kitsie), supposent cette incitation individuelle ou collective. La gestuelle y compte autant que les incantations et les formules. La grande épreuve de réalité semble bien être la parenté et la limite de la sorcellerie, mettant l'accent sur la magie d'agression, sans que son autre volet (la sorcellerie innée) disparaisse jamais. Mais la parenté est un domaine très hétérogène et d'autre part elle n'est pas le seul, loin de là !

Un tel conditionnement du corps regarde aussi le faible ou le dominé. Lui perçoit sa position par l'angoisse du cauchemar, le choc d'une accusation ou plus insidieusement du soupcon. Mais les mécanismes en cause sont les mêmes de part et d'autre d'une contradiction.

\section{L'interaction sujet-objet dans le champ de force vitale}

\section{Définition}

La force évoquée nous apparaît maintenant localisée en un "objet" ou en une "chose" (kiloko)15, la recouvrant. Continuons à suggérer que cet objet si déterminé est social. Ne nous attachons pas plus que les Kukuya à son support extérieur, à son apparence manineste, mais plutôt à son schéma de constitution : homme animalisé par exemple ou animal humanisé, à la fois dans sa logique des choses et dans sa logique des mots.

150n doit distinguer kilòkò, la sorcellerie et kilókò, la chose. 
En même temps qu'on fabrique un objet social, signifié par le buti dans son lien à l'invisible, "la personne qui le prend, subit une excitation et voit se transformer son corps". Le parallélisme des deux séquences, n'est-il-pas à chercher dans la position cachée d'un sujet social, figuré par le nouveau détenteur ? N'y a-t-il pas, à travers I'excitation, une mise en état de ce sujet, "détourné" lui-même par la force?

Tout le mouvement de "prise" du buti chez un spécialiste serait à saisir comme interaction sujet-objet "déviée" de la force sociale symbolisée, caractérisant. son acteur. S'insérant dans le cours naturel par toute une série de métonymies animales, végétales ou autres pour en faire surgir le poids de ses métaphores face à autrui (P. Bonnafé, 1970: 159-192) : lion, arbre gigantesque, fleuve sans frein. Ce détenteur de puissance, à nous de découvrir à chaque fois quelle "déviation" le traverse, ce qu'un sociologue pourrait traduire : quels groupes le socialisent et quels groupes sont socialisés par lui d'une manière principale ou secondaire.

Un chef de lignage ou de hameau, détenteur de buti, manierait ainsi comme objet caché son rapport social à ses dépendants : cadets, femmes, et réciproquement. Mais le même personnage agirait comme sujet du rapport social à ses dépendants (ce qui le rend maintenant tributaire des formes et non plus des forces) et inversement. Cette loi entrâ̂nerait, malgré les apparences, l'opposition entre réel et imaginaire. Ainsi de cet autel nkiini, protégeant le hameau, dont il enferme les membres comme une amande enclot ses graines.

\section{Formes et forces}

Mettre en relation des formes et des forces suppose qu'on ait résolu leur attribution respective. Pendant longtemps, je ne donnais à cette question qu'une réponse empirique. D'une part, les gens de la société proposaient un découpage significatif de leurs groupes. D'autre part, la sociologie pouvait tenter de construire le sien.

Je retiendrai d'abord le premier moment. Le propre d'un fait social, à la différence d'un atome physique ou d'une cellule biologique, est qu'il est déjà symbolisé. Le langage est une donnée 
interne de sa constitution. Mais c'est en avançant dans le second que j'ai pu considérer le premier. Il m'a semblé inconcevable de définir une force sociale sans affirmer aussitôt qu'elle était représentée par une forme ${ }^{16}$.

Dès lors, j'ai décrit la hiérarchie inégale de trois systèmes: domestique, lignager, marchand extérieur ou capitaliste, avec leurs rapports de production et de socialisation plus large en même temps que leurs systèmes formels. L'opération n'était ni moins, ni plus difficile que son premier temps : on ne voit pas pourquoi elle aurait été moins valide ! En effet, la liaison entre les deux m 'était donnée par un élément de liaison théorique. En retour, elle devrait me permettre d'intégrer de manière beaucoup plus satisfaisante le découpage social des habitants.

\section{Définition d'une forme sociale}

Une forme sociale, en tant qu'elle appartient à un discours social relevant du langage, se décompose en signifiant et signifié (Jakobson R., 1973). Le premier système inscrit le marquage matériel du son dans les mots et marque les effets inconscients du "sujet" dans le discours. Le second système y encode la formation de l'idée dans les signes et les effets conscients d'"objets" dans le discours. Son et idée se heurtent pour créer la signification. Notre pari est qu'on peut étendre la théorie du "sujet" individuel à son pôle collectif, parce que de toute manière, on s'est toujours trouvé dans le social. La théorie du pôle individuel n'aurait été alors qu'une première pièce d'un dispositif plus vaste, sans qu'on enregistre entre eux de rupture.

Ces deux mouvements opposés du sens ne se discernent-ils pas dans notre évocation? D'un côté, les signifiants attachés au buti représenteraient la production de la catégorie des ainés et celle en leur sein de la corporation des spécialistes ngàà face aux femmes

16Les gens qui pensent procéder autrement, par exemple en définissant un fait technique ou économique pur, méconnaissent qu'ils s'appuient sur un système de signifiants spécifiques, dont ils devraient circonscrire la validité. Arrive un moment où il faut bien rendre la parole à des paysans muets ! 
et aux dépendants. Ses effets de symbolisation entraineraient la "déviation" des sujets de part et d'autre de la contradiction des groupes. "Composition" et "excitation" résulteraient de cette structuration inconsciente.

De l'autre côté, les signifiés du buti renverraient aux principales manières de socialiser ces groupes objectivement : technique, économie, modes de socialisation culturelle, tout ce qui peut matérialiser ces groupes. Cet ensemble amènerait cette fois le "détournement" d'un objet perçu consciemment, toujours de part et d'autre de la contradiction. Le champ d'un rite serait à analyser ainsi avec les composantes objectives de ses acteurs.

Les deux mouvements viendraient s'articuler dans le discours social propre au buti, dans la mesure où il n'est pas le tout de la société, mais son idéologie dominante, renvoyant à un même rapport de forces. Pourquoi la force sociale subit-elle forcément une translation ? La socialisation provient soit de la nature (écologie, énergies), soit de la technique, soit d'une énergie interne. Le point commun de ces faits est de se fonder sur des contradictions propres. Ne peut-on penser que le type de contradictions engendre à chaque fois la translation ?

Tout cela serait formalisé à des degrés divers dans notre cas. Les spécialistes de magie sont placés à un point précis de redistribution du surtravail extorqué ou extrait. La fragmentation de leur savoir est une conséquence globale du champ social atomisant leur pouvoir. La production de groupes sociaux serait un mouvement aussi "détourné" que les objets magiques eux-mêmes ! Aucune terreur imaginaire ne peut s'établir sans masque.

\section{Le grand Sujet d'une forme spécifique}

Nous appuyant sur une étude antérieure (Bonnafé, 1978: 133-144, d'après I. Althusser, 1966), nous rappelons que le couple central de la sorcellerie vécue : sorcier-victime, exige d'être réduit, dès lors qu'on a pris connaissance du sujet $S$ d'une idéologie propre. Cette réduction ramène à la translation précédente de la force. "Ce grand Sujet a la propriété spécifique de constituer certains 
individus en sujets idéologiques dans un couple fondamental accuséaccusateur, éventuellement réversible" (Bonnafé, 1978: 136). Nous insistions sur son support : la lutte particulière entre couches et catégories sociales. Cette configuration peut venir englober celle de la magie d'agression, dont elle est la limite. On passe de l'une à l'autre par croisement. La zone du buti évoque le cycle des appropriations naturelles et son annexion par. une catégorie spécialisée. Le buti vient constituer certaines personnes en sujets spécialisés d'un savoir agissant, face à d'autres sujets qui en sont dépourvus. Sa force sociale précise a bien l'air de produire à la lettre son champ de "composition". Une superbe illustration : le système tributaire ancien produit des seigneurs sacralisés du ciel, dont la force mpu, échappe à toute accusation de sorcellerie.

\section{CHAMP}

Autant qu'à la "cuisine magique", on pourrait penser à une "chimie" ou une "physique" simples, à condition d'ajouter qu'elle reste inter-humaine, "botanique" et "zoologique". Il s'agit en effet de cerner tout un champ à présent, d'en suivre l'évolution, de le rapporter au temps et à l'espace, d'en repérer les zones homogènes et les ruptures. Les habitants ont quand même une estimation en grandeur. Imaginons donc un peu ce laboratoire de forces socionaturelles !

Dans le système évoqué au début, le champ dépendait des forces élémentaires mpini, tout autant que des forces composées mpiini. Ensuite, la localisation du buti en objet a conféré une certaine individualité aux champs partiels. Enfin, avec tout le champ interactif, la particule de départ mpini s'évanouit presque : à une extrémité, la faiblesse d'un accusé détruit sa personne, à l'autre, la force composée d'un vieil homme très puissant finit par chasser sa force originaire (mpini, mumpuki). 
D'après leur continuité et selon leur nature, les différents champs peuvent aussi se classifier. Certaines forces sont plus proprement lignagères ou résidentielles. On en isolera d'autres, tributaires avec les seigneurs de la terre et du ciel : les bikini. Le champ des premiers est plus proche des lignages; celui des seconds fait intervenir des forces s'appuyant sur des mati initiaux et des forces ancestrales avec une sacralisation en pouvoir mpu, cherchant à faire oublier son point de départ.

quand cette "violence naturelle" s'est déclenchée à son apogée, elle a donné l'impression que l'essentiel n'était pas dans les éléments sociaux, mais entre eux. Après tout, ne serait-ce pas une bonne caractérisation du champ?

\section{A. Le champ des mati}

1. Continuité globale du champ

La séparation qui règne dans la vie économique, les communications difficiles et un certain cloisonnement culturel pourraient mener à conclure : le champ des mati reflète cette discontinuité. Du point de vue de la croyance, c'est tout le contraire ! L'homogénéité des éléments est si forte, leur intensité si parallèle partout, qu'à peine si on note un petit décrochage avec le bourg sous-préfectoral. "Je ne crois que ce que je pense pouvoir faire croire", dit magnifiquement J.-T. Desanti (1982: 36). Tout le mécanisme de la croyance s'investit dans I'univers évoqué. Même avec des gens jamais fréquentés, dans de petits cercles interseccants, une osmose s'établit et gagne le pays entier, fonctionnaires et scolarisés compris, même s'ils ont acquis du retrait. Une telle continuité répète la même contradiction.

\section{Son contenu symbolique}

Pouvons-nous préciser le contenu symbolique entrevu à travers les forces du champ? Il est sûr qu'on ne rencontre pas n'importe quelle composante. Parmi les principales, j'en citerai quatre : vieux-jeune, homme-femme, chef de hameau-subordonné, chef de lignage- 
subordonné17, les premiers termes l'emportant sur les seconds. En y adjoignant un échelon supérieur : chef tributaire ou administratifsubordonné et en négligeant puissants et spécialistes ngàà déjà situés, on a un noyau formel satisfaisant. Il est probable qu'il entretient un lien étroit avec la nature du $S$ idéologique, présenté plus haut. Risquons-nous à prévoir, comme les astronomes l'ont fait des planètes, que les effets signifiants de la socialisation du champ, ont toute chance de se trouver là ! L'hypothèse se vérifie assez dans les signifiés. Comme on le verra bientôt dans l'affaire du "boa de semence", ces cinq relations drainent une grande part des hiérarchies sociales.

\section{Signification du "détour"}

Cette force des mati, nous avions voulu la saisir directement par refus d'un arrière-monde "fétichisé" et voilà que nous avons été conduit à n'en faire sans cesse qu'une saisie indirecte, "déviée". Nous avons dû admettre la nécessité d'un "ailleurs" propre à sa socialisation, puisque nous ne pouvions nous représenter la force sociale, considérée tant par les habitants que par nous18, que comme transformée. Nous n'atteignions son sujet que décentré et son objet que déplacé à travers le discours social. Etait-ce un prodige ou un mystère ? Nous sommes trop incrédule pour en convenir! C'était plutôt le mouvement des contradictions observables.

Mais, comme on l'a affirmé, il ne se cache pas plus de sujet dans une société que dans sa propre reproduction sociale (Terray, 1977: 140), qui ne reproduit que des contradictions. Il me semble que cette vue, en bonne rigueur, conduit à dire qu'elle n'a pas d'objet non plus. Oui et non, répondrai-je. Les mati représentent bien un lieu d'irréductible, tant comme objet que comme sujet (comme dans la sorcellerie). Mais leur Sujet signifiant entraine bien des sujets sociaux, leurs détenteurs par exemple, et leur objet signifié

17Les deux dernières relations, dans l'ordre inverse, dominent les deux précédentes, qui ne s'établissent pas directement, une fois de plus !

18D'après nous, la sociologie ne risque pas de découvrir un fait qui n'a laissé aucune trace dans les signifiants d'une société. 
amène bien aussi des objets sociaux, leurs diverses puissances. Une double "déviation" engendre l'interaction. Cela ne devrait-il pas conduire à une position différente par rapport à la contradiction elle-même ? Nul ne pourrait prétendre s'y placer pour énoncer la vérité, mais on ne pourrait qu'analyser l'inconscient social qui la reproduit, ce que les Kukuya ne nous ont pas attendu pour faire à leur manière.

\section{B. Le registre des effets et des preuves (sorcellerie, magie, religion et savoir faire) :}

1. L'exemple du "boa de semence" : mbomo a sala

Une femme dans la Terre Lage en 1965 avait un "boa de richesse", hérité de son lignage utérin (frère ou mère), autre nom de ce buti qui était fait pour enrichir. Ce boa volait la nuit les semences de ses "voisines" (terme pour désigner ses compagnes de travail). La femme en effet était "jalouse" d'elles (mpfuna) et aspergeait la nuit toujours leurs semences avec une eau maléfique.

Les devins consultés dévoilèrent qu'elle avait du pfuna, un mauvais génie mupfu placé dans un cabri et un boa de richesse. (On voit le mécanisme en action : le boa dérobe les semences et les ramène dans le sachet de sa mâtresse. Le cabri vient les manger. La force innée pfuna renforce la "composition"). Ils conseillèrent de faire un "appel" (kibila) en présence du seigneur de la Terre. Et si le mal continuait, alors il faudrait menacer la femme de convoquer un vrai ngàà pour la dévoiler.

Les protestations étaient parties d'une autre paysanne, sa voisine, qui se plaignait de son "défaut" (kiwena) de semences. Parcours classique : $d u$ père au père du père à l'oncle utérin de la femme lésée. Ce dernier - qui était aussi son chef de lignage vint faire sur son champ un rituel mukwu a sala : "conjuration des semences". Cendre lifuru, plantes liyuka et mutsitsima pour raisonner la sorcière, débris de la tige de palmiers lilieme. La femme lésée creuse un sillon (savoir-faire réel) dans un coin du champ. L'oncle étale la cendre et les autres plantes dessus, plante quelques graines 
d'arachides ou manioc en disant : "Que tes arachides prospèrent cette année ! Si je suis ton seul oncle, que ma parole aie tout son tranchant (ndaka me $l i$ to) ! ... Je n'ai aucune dispute te concernant..." (Là, on voit plutôt l'appel aux causes finales : l'union des ancêtres faisant celle des vivants et assurant leur succès). Mais il y a aussi la lutte pour réfréner le pfuna inné de la sorcière et la contre-sorcellerie contre son buti. Si elle ne se retire pas, alors on fera des rites redoutables (kibruki mba), etc.

Toute cette histoire, je l'ai contée surtout pour sa fin. L'oncle utérin et les parents paternels décident de monter un véritable piège avec un breuvage kitsie, qu'on placera sur le champ de la femme lésée. La sorcière, si elle ne peut se contenir, viendra boire cette eau sous forme de boa ou de cabri et absorbera ainsi un poison.

Pourquoi aura-t-elle été irrésistiblement attirée par cette eau ? Parce que la femme lésée aura pris la précaution de s'en servir pour se laver, y mêlant sa propre odeur. Elle asperge aussi son champ d'un peu de cette eau.

Le boa ou le cabri de la sorcière ont aussi une odeur. Elle va se mêler à toute une châ̂ne que nous découvrons. La cultivatrice du champ en travaillant ses arachides à la houe atteint "le boa qui est venu dans la houe et en elle-même". Car "les semences proviennent de la personne et de son mpolo".

Ce qui est central est par conséquent l'ensemble lié : femmehoue-semences-eau imprégnée d'odeur, auquel on ajoutera la terre. Tel est l'enjeu de la bataille qui s'y livre.

\section{Interprétation}

Quel bel exemple d'imbrication des causes ! De la "chose" présente dans le corps ("witchcraft") à l'efficience d'un dispositif manipulé ("sorcery") à la finalité ancestrale jusqu'à la formalisation d'un savoir-faire technique (J. Lacan, 1966: 862-877)19.

19La référence de J. Lacan aux quatre causes d'Aristote est très éclairante, à condition de n'y voir que de grandes directions. 
Quant à l'ensemble retenu comme un fascinant enjeu : femme, houe, odeur, il repose sur un lien caché aux forces de l'univers. On rencontre une séparation visible entre humains et nature (diurne) et une fusion invisible (nocturne). Sa cause en est - ou son signal ou sa loi - une liaison entre outil-corps féminin-semences, où le succès agricole (mpolo) est d'abord placé dans l'énergie humaine de la femme : ce qui est tout à fait exact. Or, comme par hasard, c'est ce lieu technique que le rapport social ou force vient investir avec ses mati.

De telles perspectives nous rapprochent de la notion de langage agissant, présente dans ndaka : "mot", "parole", mais aussi "événement", "affaire", "malheur", "accident". En de tels points, le langage lui-même devient fusionnel.

Le maintien de cette structure renvoie à I'histoire. Les femmes sont toujours confinées dans leur domesticité vivrière, les hommes s'emparant des cultures de rente et des quelques salaires. Telle est "la place" (mbuka) que les aînés viennent "régler" (kiyilika), mais parce qu'ils l'ont d'abord activée.

\section{Retour aux champs des mati}

Un document concis nous le restituera : le récit par un magicien, contre-sorcier et guérisseur de folie, de ses trois voyages au pays des morts durant son initiation à la magie. Son mấtre appartenait au peuple teke-lali (Lali) voisin, c'est pourquoi les mati vont se mêler au village des morts. Nous interrogerons la singularité avec des catégories aussi universelles que possible.

1. Le déni du réel

Nous retient ce qui découvre sa perception du champ. Accompagnant son maître une première fois chez lui à Lali, il dort en sa compagnie et l'autre disparât à sa vue : il tressaille de terreur. Son maître le fait errer auprès des tombes : sa spécialité... Plusieurs fois, il y a la vision de sorciers et d'ancêtres, "excités" par ceux qui se servent de leurs "formes" (kimpfa) pour 
tromper leurs victimes. Peu après, il voit un vrai village invisible de morts détournés. Il arrive que ces génies reprennent leurs formes animales pour accomplir des actes quasi-humains : déchausser ses sabots pour boire, comme le fait une antilope. Il rencontre aussi des villages d'animaux rangés par espèces. A son retour d'initiation, il traverse seul des plaines vides et pénètre dans une grande forêt remplie de bêtes féroces. Il marche plusieurs jours sans trouver d'issue. Un coq le remet sur la piste en lui démontrant (loin de l'anthropocentrisme) la supériorité des animaux sur les humains : Iui, au moins, connâ̂t le temps. Après plusieurs pertes de conscience, il voit clair de nouveau et revient à son village.

2. Le déni de la mort (et du sexe)

L'apprenti se demande sans cesse : "cet homme, mon mâtre, me laissera-t-il vivre? Comment survivre à tout ce que je suis en train de voir ?" A présent, il sait l'existence de cette meute de personnes retirées de la vie, mais pourtant non mortes : "...Nous nous sommes mis en route pour le village des morts. Arrivés dans une étendue déserte, mon mâtre a sifflé et tout un village s'est montré, non loin du sien. J'ai vu moi-même des gens revenant de la chasse avec leurs filets, des pêcheurs rentrant avec leurs poissons. ol fuir? Je ne suis pas sorcier. Je n'attends plus qu'une chose : la mort". L'histoire de son initiation est traversée par l'obligation permanente de respecter l'interdit de son mâtre : refuser toutes les belles femmes, qu'il lui envoie pour le tenter.

\section{Le déni de la toute-puissance}

La toute-puissance concerne le pouvoir sur la vie. C'est la grande contradiction de s'être trouvé placé à l'un de ses degrés sans pouvoir s'en servir légitimement (la perversion est prêtée aux sorciers s'accommodant de tous les déguisements et truquant sans fin le jeu des apparences). Tous les conseils de son mâtre reviennent à détenir cette force immense, en n'en faisant que des usages bénéfiques. Refuser de soigner des sorciers : c'est inutile et risqué. Ne pas demander plus que le paiement d'une cure. Ne pas réclamer trop pour faire un objet buti. Ne pas oeuvrer de son art pour s'enrichir. 


\section{L'activation instrumentale}

Le déni et la fusion caractérisent aussi bien la religion que la magie d'agression. Mais cette dernière joue toujours sur une sorte de pure efficience, même si elle requiert un savoir. Au cours de l'initiation retracée se déploient de violentes joutes et rivalités entre l'initié et son mâtre. Le second triomphe d'abord, puis le premier par un retournement complet contre son "père". Le héros parachève sa victoire en dominant son vrai père, jaloux de lui. Le couple de magiciens (experts ou spécialistes) est aussi réversible que celui d'accusateur, quand bien même il s'inscrit dans une ascension de génération.

Ce champ ne témoigne pas que ses participants vivent "a priori" une moindre réalité que la nôtre. On discerne d'ailleurs à l'intérieur de leur petite société de fortes variations sur l'accès au réel. Femmes et hommes n'y sont pas placés sur le même pied. En 1965-67, la plupart des paysannes, dépourvues de mati, répondaient aux questions sur l'usage de l'eau, du feu, du bois...par des propos beaucoup plus concrets que les hommes. On pourrait opposer encore des chefs plus politiques, comme les seigneurs du ciel jadis, à des ngàà plus tournés vers le phantasme. Enfin, plus globalement, la mainmise coloniale et ses suites, en dépossédant la plupart des habitants de leur initiative politique, les a rejetés dans un monde désadapté face à l'évolution moderne, dont les "partenaires" étrangers sont souvent encore plus invisibles que leurs mauvais esprits.

La relation de ces humains à la nature s'éclaire lorsqu'on la déchiffre comme relation simultanée à leur société. A notre sens, ce sont les rapports sociaux d'exploitation ou d'autonomie qui déterminent l'aliénation propre à la magie ou à la sorcellerie. Aussi poserons-nous aussitôt comme "oublié" dans le champ des mati, un quatrième déni (après le réel, la mort et la toute-puissance), celui du surtravail féminin et autrefois celui des dépendants. N'en supporte-t-il pas l'édifice en provoquant la naissance d'un fantastique collectif, d'un imaginaire illusoire, apparaissant comme un traitement des contradictions par une couche dominante?

Perpétuels objets contre perpétuels sujets, le cadre se déplace à travers le temps. "Autrefois, dit un vieux paysan, chez nous, 
seuls les seigneurs détenaient plus de puissances que les autres; toujours, ils voulaient dominer et se faire craindre". Pius tard, ce seront les habitants d'un hameau, affrontés à leurs ainés ou protégés : marge indécise. L'organisation lignagère donne aux supérieurs peu de moyens immédiats de coercition et ils auront recours à l'arsenal dévié des forces invisibles. Ainés et cadets se heurtent sur le terrain de la production marchande, où l'individualisme fait éclater la "personne" ancienne (Dupré G., 1977).

Mais les résistances n'ont jamais fait défaut chez les dépendants, chaque couche cherchant à assurer son indépendance. Les points d'autonomie relèvent eux de l'imagination, une toute autre force souvent lente à se faire jour et qui emmagasine ses réserves en vue d'une rupture éventuelle. La sociologie critique devrait se donner les moyens de distinguer entre ces deux sphères. L'histoire lui est précieuse dans son investigation des forces et des formes. Les rapports majeurs, qui ont marqué la région, aux dires des gens, ont été l'esclavage et la dépendance tributaire, engendrant une force sociale supérieure aux systèmes lignager et domestique. Ensuite, ce fut l'Etat colonial, régissant la nouvelle économie. Tels sont les phénomènes qui nous guident, quand nous cherchons l'origine des objets mati, nés aussi dans l'organisation inférieure. A travers leur imposition, nous viserons non une "violence fondatrice", dangereuse métaphysique, qui a la vie dure 20 , mais 1 'installation précise de rapports de socialisation. Ainsi de ce seigneur du ciel, à la fin du $18 \mathrm{e}$ siècle, plantant sa lance dans le pied de son esclave pour se redresser !

Selon nos conventions, les formes représentant ces rapports sont à désigner avec la même rigueur. Sans doute, le pfuna inné de la sorcellerie renvoie-t-il à une souche archaïque que perpétuent les hameaux et lignages non marchands pour une part. La magie d'agression des mati correspondit à des élargissements successifs dans le champ, jusqu'à y inclure l'argent C.F.A. De nouveaux signifiants sont pris en considération même par des illetrés, puisqu'on trouve d'assez nombreux fous de l'écriture ou des études - ou du chômage.

20 La critique de F. Engels dans son Anti-Duhring convient encore aux thèses de R. Girard sur la violence ét le sacré. 


\section{La production du champ social des mati}

Comment la force sociale produit-elle son champ par translation21? Sa formulation la plus générale s'appellerait socialisation domestique, lignagère, marchande extérieur. La Force sociale symbolisée y serait à la fois le sujet et l'objet d'un processus de socialisation mû par son interaction qui déterminerait celle des sujets et des objets sociaux. Si l'on préfère, elle régisait les forces et les formes visibles ! La translation déterminerait son objet et son sujet.

Dans le système lignager dominant, c'est le rapport aînés-femmes qui est décisif, mais à l'intérieur d'une bio-valorisation propre du surtravail féminin, faisant intervenir le travail des cadets du secteur vivrier au secteur marchand. Celle-ci s'analyse à partir des biens de prestige ou des biens marchands. Cet objet entraîne les forces et donc les objets sociaux déplacés. L'exemple du "boa de semence" a fait apparâtre ainsi le lien entre la santé de la femme malade et son chef de lignage. Ce qui est remarquable est que le changement historique des forces va ressortir chez les sujets dans les formes : l'apparition d'un secteur commercialisable introduit dans le champ des puissances de nouvelles formes modernes (fétiches de richesse).

Cette valorisation est tout aussi décisive dans la position d'un Sujet entrainant les formes et les sujets sociaux décentrés. L'ancienne valorisation non marchande, reposant sur des unités lignagères est toujours représentée, d'où les traces de sorcellerie innée. Les nouvelles formes de "composition" des puissants accompagnent le passage à la magie d'agression. Les accusations de sorcellerie portent sur les gains de petits producteurs marchands. Le fait notable est ici que le changement fait ressortir des altérations dans les forces. On ne craint plus les mêmes choses : on redoute qu'un cadet ne se libère grâce au marché capitaliste.

21 Andréani T., 1978, définit l'ensemble du procès de travail et de production comme valorisation. Celle-ci serait la clé de la socialisation. 
L'essentiel nous apparaît à présent comme une valeur sociale d'un certain type, phénomène à la fois objectif - conscient et subjectif - inconscient. Une couche d'ainés - et des classes extérieures - exploite des femmes et se pose comme le sujet du processus, mais leur résistance rendrait sa perpétuation difficile : c'est pourquoi elle est conduite des deux côtés à un certain maniement des contradictions. Il n'y a pas d'autre objet ou sujet, en effet, que la valorisation aux trois niveaux indiqués et son caractère transformable pour des sujets et des objets sociaux, engagés sur un terrain politique.

Quant au discours social des mati, ses signifiés manifestent l'objet irréductible du réel social et ses signifiants figurent l'unité impossible de la contradiction. Les ainés, les dépendants, les femmes se situent de part et d'autre de cette inégalité. Tel est le milieu, dans lequel nous avons été conduit à plonger l'objet buti pour décrire son activation. L'opération éclaire les dénis à l'oeuvre dans le champ. Comme par hasard, le déni "oublié", celui du surtravail féminin, expliquerait pourquoi on rencontre un Surmonde, une Survie, un Surpouvoir chez des aînés, tout de même contraints de rester légitimes.

\section{E. En quoi la force, l'objet, le champ sont-ils particuliers}

1. L'énergie humaine nous a paru être au centre du travail, de la production et de la vie sociale au sens large. Son usage commande une qualification élémentaire, portant sur l'âge, et une division élémentaire des tâches, portant sur le sexe, par scission coordonnée entre deux énergies non spécialisées en permanence, sauf dans cette seule opposition. Ce cadre n'exclut pas d'autres différenciations secondaires, que les artisanats illustrent bien. Le jeu des systèmes domestique et lignager socialise ce cadre de départ, à travers lequel unités de production et de consommation demeurent homologues ( $E$. Terray, 1969: 145).

Aussi les rapports sociaux y sont-ils interpersonnels (et communautaires avant 1900). La couche d'aînés vise à contrôler 
dans ses hameaux comme dans ses lignages, une couche de dépendants socialisés sur ce mode. L'aristocratie tributaire, qui voulut rompre avec ces pratiques, échoua à créer une classe durable. Elle aussi, dut s'adapter à ce qu'elle voulait exploiter.

A la place d'une séparation de classe, on trouve ainsi un type propre de liaison-séparation à la fois entre homme et nature et entre homme et homme. La rupture coloniale, avec ses remaniements successifs, n'empêcha pas ce type de perdurer au prix de nombreux changements. Ces rapports font comprendre le caractère de la force sociale, représentée par les mati. Des blocs de filiation réelle ou fictive sont régis par des aînés dans la hiérarchie évoquée avec ses variations de contexte (les quatre relations du noyau). Il s'est toujours agi sur deux siècles de canaliser de l'énergie humaine liée à ses groupes d'entraide, à ses instruments, à son savoir, à ses ressources écologiques, à sa capacité d'association (mariage, amitié). Plutôt que de scinder (magiquement) production et reproduction sociales, mieux vaut voir comme elles sont identiques à tous les échelons. C'est cet objet contradictoire qui est surtout représenté par le Sujet de la sorcellerie, forme des contradictions existantes.

La magie d'agression dominante marque un moment de construction plus sophistiqué. Le chef ne règne plus sur son seul groupe, souvent non marchand (sorcellerie innée), mais la production englobe d'autres éléments, très souvent marchands. Un quadrillage plus compliqué requiert des spécialistes ngàà. Mais, quel que soit le cas, le corps est le siège privilégié de la force sociale. Comment saisir sinon, dans la lettre des agissements, qu'un puissant s'empare d'une énergie de crapaud afin de mouvoir une force humaine contre une autre ?

Ce lot de pratiques ne doit pas être vu uniquement comme phantasmatique, comme semble le suggérer E. Evans-Pritchard à propos des Nuer (1940), la magie répondant à toute question locale sans se soucier du réel plus vaste. Arbitrage, règlement des conflits, contrôle des accusations, connaissance des lois résidentielles et des mécanismes d'alliance sont de vrais savoirs oraux, ainsi que certaines thérapeutiques. 
2. Si les forces sociales sont contradictoires, alors leurs formes devraient pouvoir être saisies comme résultantes de conflits les représentant. L'idée occupait déjè notre ancêtre L.H. Morgan dans son projet malheureusement en friche de retracer une évolution sociale. D'après notre esquisse, la mise en évidence de vraies ruptures dans les forces sociales devrait permettre de repérer des discontinuités dans leurs formes. Le maintien des mêmes mots pouvant dissimuler une rupture réelle, l'examen ne doit pas être superficiel. Ainsi, on nomme encore aujourd'hui un individu accusé ou soupconné: ngaa-pfuna "possesseur de sorcellerie innée". Or, ce terme n'est plus mis en contraste avec la magie d'agression, parce que l'alternative ancienne a disparu, le second pôle ayant prévalu.

\section{En guise de conclusion comparative}

1. La description générale de cette force est restée limitée au social, en tentant de suivre son double mouvement contradictoire entre les mots et les choses, le langage reproduisant par son discours social la socialisation inconsciente et consciente des groupes. Voilà la démarche qui nous a permis de particulariser notre cas.

Aux habitants de ce petit plateau, nous avons emprunté leur tripartition entre force, objet, champ, qu'ils appliquent à leur magie. Je la crois très fructueuse pour notre discipline. Saisir du même concept un système de représentations, un objet local à manier, un champ différentiel de valeurs témoigne d'une belle capacité de synthèse.

2. L'évocation des buti, nous l'avons construite comme un cas particulier de force sociale. Par voie de conséquence, nous avons cherché à détecter, comme au radar, les contradictions sociales, présentes dans ces faits spécifiques. Ce sont celles-ci, qui nous ont permis d'identifier le type de forces symbolisées en jeu. Des faits d'exploitation sur les femmes et les dépendants ont abouti à la formation de zones d'imaginaire fantastique, des aînés aux experts (pour en rester à ceux du pays). Des faits d'autonomie, moins bien 
relatés sans doute, ont été créateurs de zones d'imagination sociale, au contraire. Ne sont-ils pas à la source du plaisir qu'on a pu prendre par instant à métamorphoser la nature, en symbolisant le réel d'une manière familière et riche, parfois neuve? Les résistances à l'oppression (chansons des femmes, proverbes) s'enracinent au même terreau.

Dans cette petite société, comme dans n'importe quelle autre, il existe un "au-delà de la vie sociale immédiate", un "ailleurs", où se joue le combat entre ces deux forces. Ne faut-il pas l'admettre pour échapper aux impasses entre un capitalisme et un anticapitalisme, aucun des deux ne pouvant comprendre l'attrait du religieux - par exemple islamique - pour des peuples opprimés?

3. Cet "ailleurs" ne se trouve pas dans un autre monde, mais dans l'inconscient social, représentant les contradictions de I'univers vivant des groupes. C'est pourquoi il vaut mieux $1 \dot{e}$ nommer "en-deģà de leur vie sociale immédiate". En ce sens-là, il y a de l'invisible partout.

Une première tendance est de s'orienter vers l'unité profonde d'un système de signes, comme le discours social d'un groupe ou d'une société. Leur conscient social ne peut que reproduire le même champ... On voit mal comment les signifiants sociaux feraient apparaître des propriétés introuvables dans les signifiés et dans les forces. C'est un encouragement ; s'il existe des ruptures dans une évolution sociale humaine, alors nous pouvons mettre en lumière des systèmes de signes différents à chaque phase. L'hétérogénéité traverserait notre histoire sociale, délimitant autant d'inconscients et de conscients différents. Bénéfice de plus, à I'intérieur d'une période, nous pouvons trouver une certaine homogénéité, sans ignorer que nous avons affaire à des strates d'âge différent, même dans l'instant.

Mais au coeur de la sociologie apparaît toujours le caractère contradictoire de la socialisation. Aussi devons nous privilégier la scission, la division. A quoi renvoie le champ des mati? A leur absence presque totale chez les femmes, reliée à un défaut complet 
de droits résidentiels. On devrait en déduire l'existence de signes opposés pour les aînés.

C'est par là qu'il nous convient de voir comment subsiste cette troublante impression, que rien de ce qui est social ne nous est étranger, si reculée que soit l'époque qui nous parle. Comment penser cet écart, d'un autre genre que celui séparant aujourd'hui les nantis des autres? Les deux approches s'imposent pour étudier une région du Congo!

4. Evoquer un cas comme le nôtre nécessite de faire appel aux étonnantes découvertes de Marx dans la dynamique sociale tout autant qu'à celles de Freud dans l'inconscient. Comment penser la matérialité du signifiant buti sans se soucier du sujet social? Ces résultats sont à placer à mes yeux dans la socialisation recherchée. Si incongru que cela semblera à certains, je me suis inspiré de certaines réflexions de Marx sur la valeur marchande, où il fait d'elle le "sujet d'un processus" qui "altère" son objet même (Capital, I, 1: 159). L'objectif pratique devrait être de bâtir une analyse sociale critique, capable d'aider à la prise de conscience des contradictions des groupes d'une manière moins mécaniste. Je n'ai fait qu'un tout petit pas dans cette voie.

5. En retour, quelies questions poser à notre société?

Certains de nos puissants se sont emparés aussi des choses de la nature par un "coup de force" initial (qui se recommence dans des pays $d u$ tiers monde en autant d'accumulations sauvages). Dotés de la faculté d'entreprendre ou de gérer22, ils appartiennent à une classe à part, qui a le monopole de la mise en valeur planétaire. De leurs décisions dépend le sort de millions d'hommes, qui ne sont pas dans leur secret. Pas d'autre solution pour les faibles que de s'en remettre à eux pour protections diverses. Ne sont-ils pas les seuls à connâttre les techniques prodigieusement complexes de notre nouvel environnement?

22L'énergie principale s'est déplacée du corps à l'outil automatisé dans I'histoire des valorisations. 
Comment règnent-ils? Parfois explicitement par magie. Une affiche du métro parisien offre ce matin "la magie d'avoir un compte à la B...". On ne craint plus la sorcellerie innée, mais les slogans racistes tuent aussi sûrement qu'elle. Quant à l'efficience, ne se trouve-t-elle pas à l'oeuvre dans un monde où la publicité (qui ne servirait même pas d'abord à vendre) est censée façonner les consommateurs comme n'importe quelle propagande d'Etat totalitaire (qui ne servirait même pas d'abord à persuader)? Le Chef de l'Etat ou le Patron d'entreprise apparât toujours en personne ̀̀ un moment de son culte, incarnant les causes finales : ordre et désordre. La religion des Sciences complète le tableau dans un sens ou dans l'autre.

Un système de représentations des forces naturelles et sociales (droit d'investir, contrôle des énergies et des technologies, écart inouì et métaphysique entre le haut et le bas), des localisations de forces-objets dans un espace désarticulé (médias représentant les entreprises, les administrations), un champ de valeurs multinational, dominé par les liens capitalistes.

Là où les contradictions sociales sont indépassables, bloquées, là, dans ces poches d'exploitation, ont toute chance de se trouver les plaques d'imaginaire illusoire, déréalisant notre univers. Notre "âme" matérielle ou spirituelle peut n'être rien d'autre qu'une retombée de "Lesieur" ou de "Nestlé" (la force de I'huile et du lait) ou de l'esprit du Grand Chef génial, au-dessus de nous.

"Ni le soleil, ni la mort ne peuvent se regarder en face", disaient les anciens Grecs. De même le mouvement d'une contradiction sociale ne pourrait se figurer directement : image illusoire ou fulguration soudaine. C'est pourquoi notre magicien, Ngalikie, après ses trois voyages au pays des morts durant son initiation, se demandait: "comment vivre après tout ce que j'ai vu?". Cet en deçà du social est toujours à conquérir par l'imagination contre le phantasme, c'est-à-dire par l'autonomie contre l'exploitation. Vivons-nous pour nous-mêmes de l'individuel au collectif ou vivonsnous pour un autrui oppresseur?

\section{Pierre Bonnafé}

Laboratoire de Géographie et de Sociologie comparée - CNRS 


\section{Bibliographie}

Althusser L.

1966 "Idéologie et appareil idéologiques d'Etat" (note pour une recherche), La Pensée, Paris.

Andréani T.

1978 Propriété, Direction, Possession. Manuscrit inédit.

Augé $M$.

1975 a "Les Parents et les autres" et "Les Parents et le reste" in Les domaines de la Parenté, Filiation, Alliance, Résidence, Paris

1975b Théorie des pouvoirs et idéologie, Paris, Hermann.

Balibar E. et Althusser L.

1968 Lire le Capital, Paris, Maspero.

Benveniste $\mathrm{E}$.

1966 Problèmes de linguistique, Paris, Gallimard.

Desanti J.T.

1982 Un destin philosophique ou les pièges de la croyance, Paris, Poche.

Jakobson $\mathrm{R}$.

1973 Questions de Poétique, Paris, Minuit.

1976 Six leçons sur le son et le sens, Paris, Minuit.

Dupré G.

1977 "Sorcellerie et salariat" in Les Temps Modernes, Paris

Dupré M.C.

1984 Naissances et renaissances du masque "kidumu". Art, politique et histoire chez les Teke Tsaayi, Thèse d'Etat, Paris V.

Einstein A. et Infeld L.

1983 L'évolution des idées en physique, Paris, Flammarion.

Evans-Pritchard E.E.

1937 Witchcraft, oracles and magic among the Azandé, Oxford.

Foucauld M.

1970 Les mots et les choses, Paris, Gallimard.

Lacan J.

1966 Ecrits, Paris, Seuil. 
Lucrèce

1984 De la nature des choses, Paris, Belles-Lettres.

$\operatorname{Marx} K$.

1950 Le Capital, Ed. sociales.

Terray E.

1969 Le marxisme dans les sociétés primitives, Paris, Maspero.

1977 "De l'exploitation" in Dialectiques $n^{\circ} 21$, Paris.

1978 "L'idéologie et la contradiction. A propos des travaux de M. Augé", in L'Homme, juillet-décembre, XVIII: 3-4.

Vansina $\mathrm{J}$.

1973 The tio Kingdom of the middle Congo, 1888-1892, 0xford.

Certains de mes travaux prolongent les descriptions :

Bonnafé P.

1970 "Objet magique, sorcellerie, fétichisme?" in Nouvelle Revue de psychanalyse (numéro d'automne).

1973 "Une grand fête de la vie et de la mort : le miyali des Kukuya" in L'Homme (numéro de juin).

1978 Le lignage de la mort, Nzo lipfu, Paris, Nanterre. 